\title{
A Choice of Lower Complexity for Two Filtering Operations Based on F-OFDM
}

\author{
$\mathrm{Li}$, Xianru ${ }^{1, a}$, Huang, Wei $\mathrm{i}^{2, \mathrm{~b}^{*}}$ and Zhao, Yixin ${ }^{3, \mathrm{c}}$ \\ ${ }^{1}$ School of Computer and Information Science, Southwest University, Chongqing, China \\ ${ }^{2}$ School of Computer and Information Science, Southwest University, Chongqing, China \\ ${ }^{3}$ School of Computer and Information Science, Southwest University, Chongqing, China

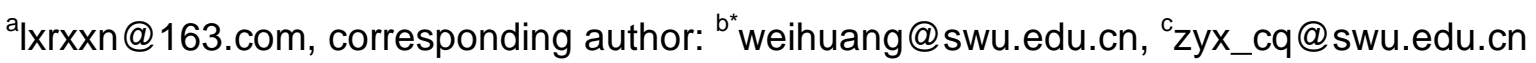

\begin{abstract}
Keywords: filter complexity; linear convolution; fast convolution; F-OFDM
Abstract. This paper introduces a choice of lower complexity for two different filtering operations based on Filtered OFDM (F-OFDM). The first filtering operation is implemented by linear convolution in time domain, and the second one is realized by multiplication in frequency domain (fast convolution). They are applied to guarantee a good performance for different data lengths. In order to always get the lower complexity of the filtering operation for F-OFDM, this paper combines both of them, and proposes a new filtering operation, which can ensure a good performance for larger class of data with almost all kinds of lengths. By comparing the computational complexity of two kinds of filtering operations and considering the effective calculating length (space complexity) of the filtering operation in frequency domain, the lower complexity of filtering operation is chosen according to the data length based on F-OFDM. Simulation based on F-OFDM in this paper illustrates the lower computational complexity of the filtering operation.
\end{abstract}

\section{Introduction}

The emergence of 5G technology and ICT network indicates the arrival of the next generation of global connected digital society. 5G supports rich business scenarios, each demands the different waveform parameters according to the business scene. Waveform is the most basic technology in the physical layer of wireless communication. The conventional waveform (CP-OFDM) can't meet the demands of the future $5 \mathrm{G}$ any more owing to its own shortcomings.

CP-OFDM waveform has been widely used in many communication domains, such as LTE 4G, WIFI and other wireless communication systems, as its high transmission efficiency, simple implementation, easy to combine with MIMO etc. CP-OFDM has its own disadvantages, however, high side lobes in frequency and high peak-to-average power ratio (PAPR) are included. The former is still treated as a drawback, while the latter has been lightened in LTE using discrete Fourier transform-spread OFDMA in the uplink [1]. Filtering has already been considered as a solution to the high side lobes of the conventional CP-OFDM $[2,3]$.

In order to meet the demands of the next waveform for future $5 \mathrm{G}$ system, relatively wide $5 \mathrm{G}$ research activities have proposed several new waveforms. UFMC was proposed in [4,5] based on sub-band filtering of ZP-OFDM. Frequency localization of UMFC is much better than conventional CP-OFDM, but the filter length of UMFC is limited within its ZP length, this leads to its performance is not good as some other new waveforms (such as F-OFDM, FBMC). FBMC was put forward by 5GNOW project [6], good spectrum localization for each subcarrier is provided by FBMC schemes, but there seems no need to do so, since the allocation of modulation and resource takes blocks as a basic unit usually.

Low side lobes in frequency and can combine advantages of conventional CP-OFDM is the inevitable requirement of 5G based waveform [7]. F-OFDM was raised in [1] based on the sub band filtering of OFDM, the system bandwidth is divided into several sub bands, there is only a very low bandwidth overhead, and the waveform parameters of each seed belt are different according to the actual demand. All the sub bands are filtered through filter to realize the decoupling of each sub band 
waveform. F-OFDM takes OFDM as its core waveform. This property is a pregnant superiority over OFDM-OQAM [8]. What's more, the performance of ISI and ICI in F-OFDM much better than OFDM-OQAM. The main difference of UFMC and F-OFDM is that the filter length of the latter can exceed the CP length. On the contrary, filter length of UFMC is limited within the ZP length. Moreover, a better balance between frequency and time localization can be achieved by F-OFDM [1]. Stated thus, F-OFDM is more able to meet the needs of 5G waveform technology than the others. So this paper introduces a choice of lower complexity for two different filtering operations based on F-OFDM.

Next in Section II, two problems needed to take into account about the complexity of filtering operation are stated in turn. In Section III, the solutions corresponding to the problems mentioned in section II are described. The lower computational complexities of simulation are illustrated in Section IV. The conclusions about low side lobes of F-OFDM and the lower complexity of filtering operation are presented in Section V.

\section{Problem Description}

F-OFDM retains numerous characteristics of OFDM. On the other hand, F-OFDM not only inherits high computational complexity of OFDM but also introduces the complexity of the filtering operation.

Conceptually, F-OFDM filtering operation is usually achieved by linear convolution in the time domain. Assuming $f h[n]$ is the response of the filter in time domain, and $f L$ is the value of its length. $i n[n]$ represents the primary data to be filtered, then the filtering operation in time domain can be expressed as follows:

$$
o[n]=\sum_{m=0}^{f L-1} i n[n-m] f h[m]
$$

This filtering operation in time domain owns high computational complexity when $\operatorname{in}[n]$ or $f h[n]$ is large.

Meanwhile, F-OFDM filtering operation may also be realized in frequency domain by fast-convolution, depending on the basis of the multiplication in frequency domain is equivalent to the circular convolution in time domain. That is to say, the value of frequency domain of the primary data and the frequency response of the filter need to be acquired first. After that, multiplication will occur between the two operands which are transformed in the previous step. Finally, the result achieved in second step is transformed to the time domain again. The data to be transformed to frequency domain should be segmented from the primary data in general. This process is described as follows:

$$
o_{i}[n]=I F F T_{L+f L-1}\left\{F F T_{L+f L-1}\left\{i n_{i}[n]\right\} F F T_{L+f L-1}\{f h[n]\}\right\}
$$

The meaning of $L$ is the length of segmented data to be transformed to frequency domain. The $i_{i}[n]$ is one of the data segmented from $\operatorname{in}[n]$. The $o_{i}[n]$ contain the finally filtered result of the corresponding $\operatorname{in}_{i}[n]$.

In formula (2), the result of $o_{i}[n]$ is equivalent to the circular convolution of $i n_{i}[n]$ and $f n[h]$ in time domain. The first $f L-1$ data of $o_{i}[n]$ is the last $f L-1$ data of $i n_{i}[n]$, the rest data of the $o_{i}[n]$ is equal to the linear convolution of $i n_{i}[n]$ and $f n[h]$ in time domain. So how to deal with the circular convolution produced by multiplication in frequency domain becomes the first consideration when the filtering operation in frequency domain is implemented. Moreover, formula (2) (filtering operation in frequency domain) has less computational complexity but more space complexity than formula (1) (filtering operation in time domain) when $i n[n]$ or $f h[n]$ is large enough. Therefore, which filtering 
operation is to be chosen by comparing the two different filtering operations is the second problem should be considered.

\section{Solutions To The Problems}

Overlap-Save (OLS) Method [9] can be used to solve the problem of circular convolution produced by formula (2). As shown in 1.a of Figure 1, $f L-1$ zeros before the primary data are added, then to cut the primary data short to the length of $L+f L-1$. This meaning is that each segmented data's length is $L+f L-1$, and two consecutive segmented data have $f L-1$ overlapping data. The selection of $L$ depends on a concrete realization of filter in F-OFDM. In principle, $L$ should make the $L+f L-1$ as the integer power of 2 to facilitate the FFT/IFFT transformation. After the primary data segmented, the FFT transform is carried out by $L+f L-1$ points for the every segmented data. The result is multiplied by the frequency response of filter, which is pre-computed in the early stage. And then to transform the result obtained in the previous step to the time domain. This process is shown in 1.b of Figure 1. The calculative results of 1.b of Figure 1, the first $f L-1$ data of the $o_{i}[n]$ contains the last $f L-1$ data of $i n_{i}[n]$, the rest data of $o_{i}[n]$ is the result of linear convolution of $i n_{i}[n]$ and $f h[n]$. Thus, just as show in 1.c of Figure 1, the first $f L-1$ data of $o_{i}[n]$ should be discarded before the final results are obtained.
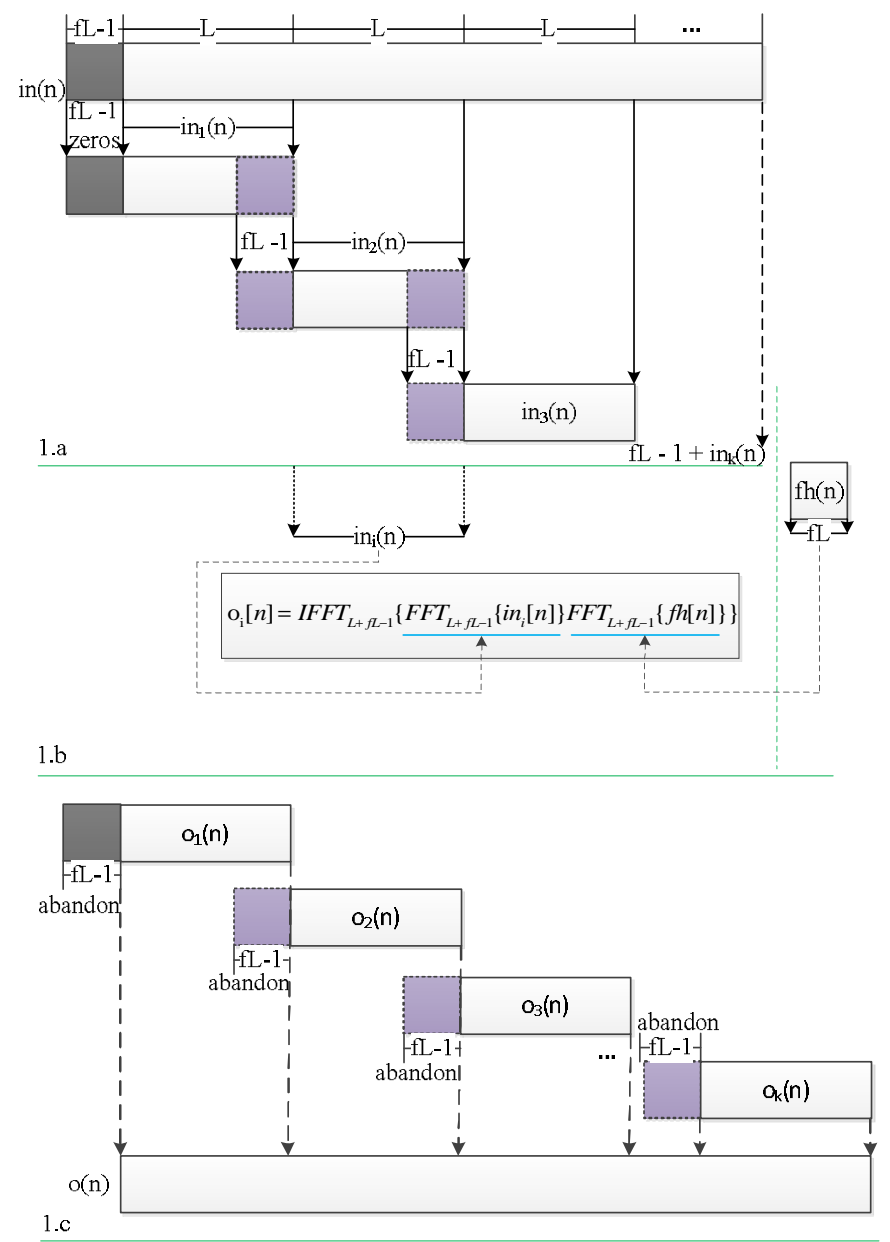

Fig 1. The process of filtering operation in frequency domain

The segmentation of $i n[n]$ is the key to analyze the complexity of the two different filtering operations. If the $d L$ is the length of the primary data and the additions of filtering operation are omitted, the total number of complex multiplication in formula (1) is about: 


$$
\left(\frac{d L}{L}\right) \times L \times f L
$$

The total number of complex multiplication in formula (2) is about:

$$
3 F F T_{L+f L-1}+(L+f L-1)
$$

If $f h[n]$ has been pre-computed, it costs only:

$$
2 F F T_{L+f L-1}+(L+f L-1)
$$

By expanding $F F T_{L+f L-1}$, the number of complex multiplication in formula (5) as follows:

$$
\frac{\mathrm{d} L}{L} \times\left[2(L+f L-1) \times \log _{2}{ }^{(L+f L-1)}+(L+f L-1)\right]
$$

So the ratio of the number of multiplications of filtering operation in frequency domain and time domain is achieved as below:

$$
\frac{2(L+f L-1) \times \log _{2}^{(L+f L-1)}+(L+f L-1)}{L \times f L}
$$

Formula (7) plays the role of judging the lower computational complexity of the two kinds of different filtering operations, once the length of the filter $f L$ in F-OFDM and the ratio of invalid computation $\lambda$ in frequency domain are determined. The value $\lambda$ ranges from 0 to 1 (the boundaries are not included). As a matter of fact, $\lambda$ represents the ratio of the extra storage space of invalid computation and the storage space of entire segmented data. The value of $\lambda$ is equal to $f L-1 / L+f L-1$. The ratio $\gamma$ of multiplications of filtering operation in frequency domain and time domain will be computed by formula (7), the results are illustrated in Figure 2. 


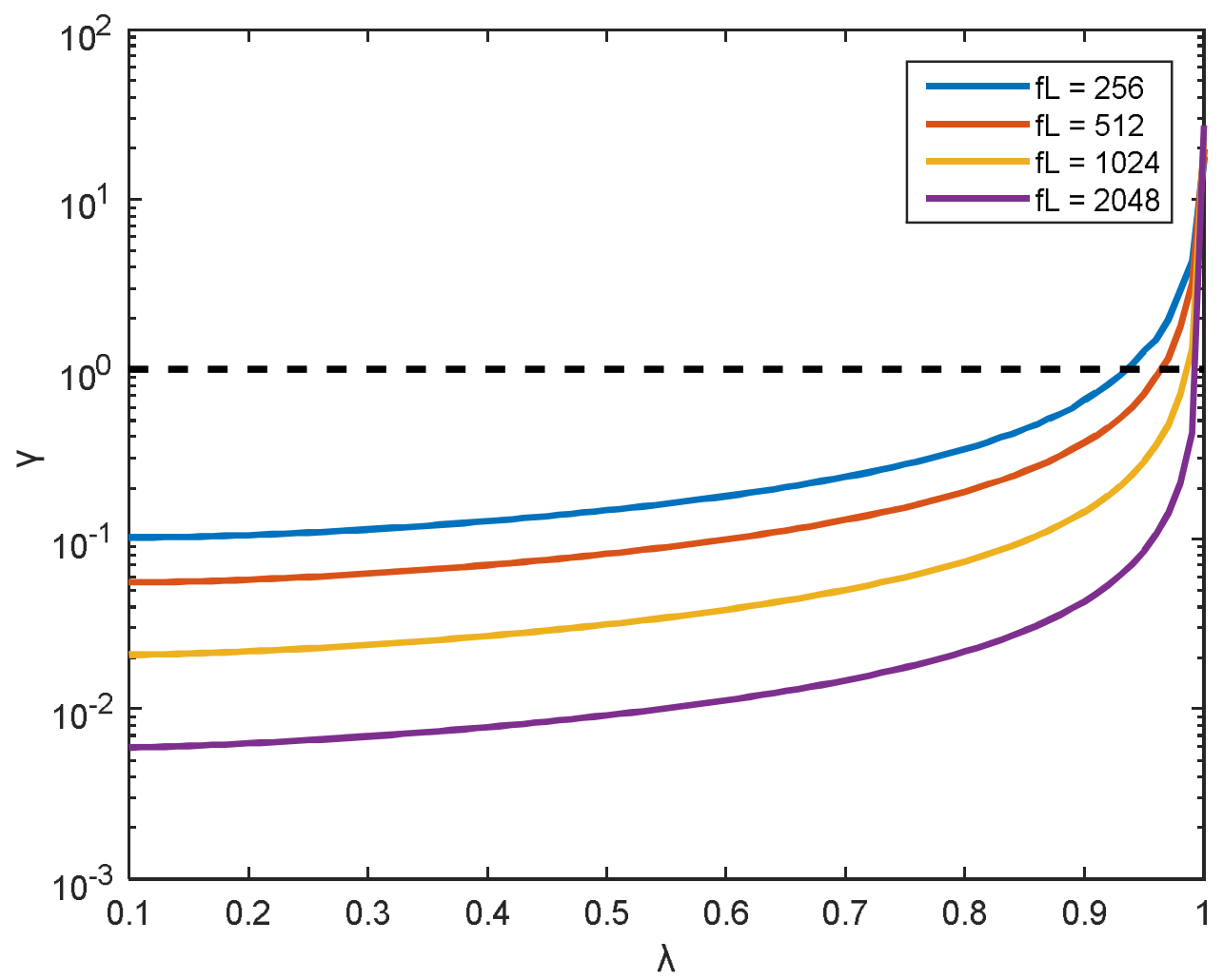

Fig 2. Ratio of multiplications of filtering operation in frequency and time domain

Three main conclusions about complexity of filtering operation can be grabbed from Figure 2. First of all, the length of filter larger is, filtering operation in frequency domain owns the better performance. There may be different sizes of filter in different sub bands of F-OFDM. Secondly, the $\lambda$ smaller (the primary data is large enough, that is to say the invalid computation is less) is, the better computational performance owned by filtering operation in frequency domain. In other words, the better computational performance and the more space complexity both owned by filtering operation in frequency domain along with the $\lambda$ increasing properly. Thirdly, the performance of filtering operation in time domain may be equal even better than frequency domain's along with the $\lambda$ going on growth.

So the better filtering operation can be chosen according to the actual requirement. There may need less computational complexity or less space complexity sometimes, and need to balance both the computational and space complexity in some other time. For instance, as the $\gamma=512$ and the $\lambda=0.8$, the computational complexity of the filtering operation in frequency domain is lower than time domain's, but another more 512 storage space of elements needed in one single sub band. The filtering operation in time domain can be chosen if the lower space complexity is more effective than the lower computational complexity at this moment.

\section{Simulation}

To make it easy to understand, downlink transmission of all subcarriers to a single terminal of F-OFDM simulated by MATLAB in this paper. Namely, this paper implicitly assumed that all F-OFDM subcarriers are transmitted from the same transmitter to a certain receiver. The simplified block diagram of F-OFDM is shown as below: 


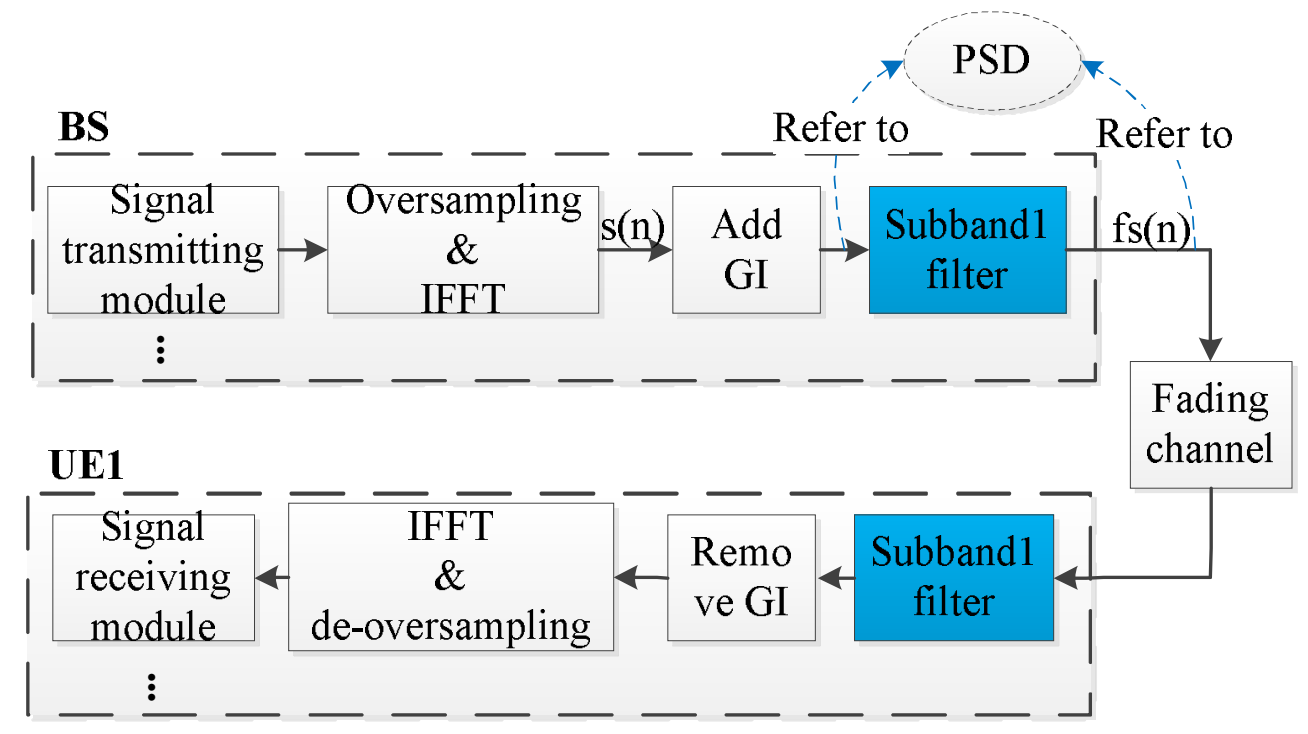

Fig 3. The simplified block diagram of F-OFDM

The block diagram of F-OFDM above is consisted by three portion: the BS(Base Station), Fading channel and the UE1(User Equipment). The "Signal transmitting module" of BS includes "Data source", "CRC", "FEC" and "modulation" as shown in Figure 4. The PSK/QAM symbols produced after the modulation block. There is oversampling present for PSK/QAM symbols, which would void introducing intolerable aliasing when passes these symbols though a DA [10]. $s(n)$ is the OFDM symbol, which is the output of inverse Fast Fourier Transform (IFFT), the inputs of IFFT are the PSK/QAM symbols. In complex baseband notation, a basic OFDM signal $s(n)$ during the time interval $m T_{\text {sym }} \leq t \leq(m+1) T_{\text {sym }}$ can be described as [11]:

$$
s(n)=\sum_{k=0}^{N_{c}-1} s_{k}(t)=\sum_{k=0}^{N_{c}-1} a_{k} e^{j 2 \pi k \Delta f t}
$$

$s_{k}(n)$ is the kth modulated subcarrier with frequency $f_{k}=k \cdot f$, and $a_{k}$ is the PSK/QAM symbol. The $m T_{\text {sym }} \leq t \leq(m+1) T_{\text {sym }}$ is the mth OFDM symbol interval. The guard interval will be added in OFDM symbols to void the ISI introduced by the delay of the fading channel. The filter of sub band is designed based on soft truncation just like described in [1]. The F-OFDM symbol is achieved after the OFDM symbol filtered by the sub band filter. The filter here contains two different filtering operations which are described in Section III. The better filtering operation would be chosen according to the length of OFDM by considering the $\gamma$ and $\lambda$.

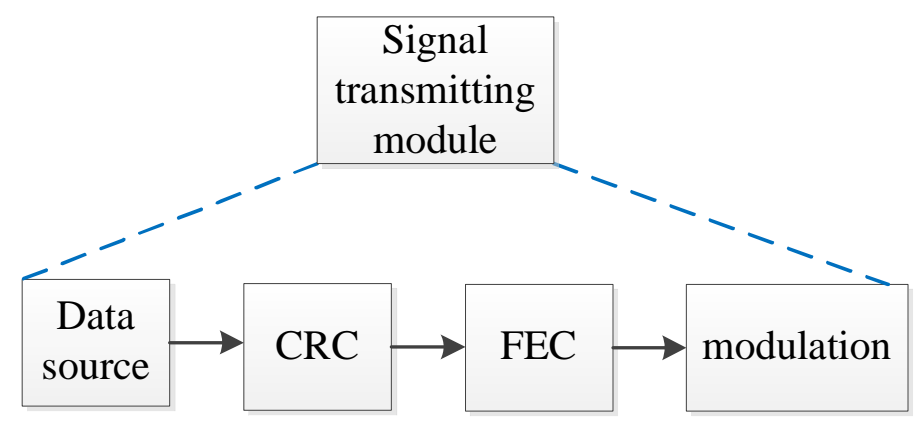

Fig 4. The contents of "Signal transmitting module"

The fading channel of the F-OFDM downlink is the typical urban channel with UE speed of $3 \mathrm{~km} / \mathrm{h}$, the transmission delay of UE is between 0 and the quarter of the interval of OFDM symbol. 
The process of the UE1 is the inverse process of the BS, the sub band filter of UE1 is corresponding with the filter of BS. Its main function is to obtain the F-OFDM symbols of UE1, which are propagated by the BS. Especially, filter of UE1 is important when there are multi users of the F-OFDM. The "demodulation", "decoding", "CRC" and "Data source" are included in the "Signal receiving module", as shown in the Figure 5.

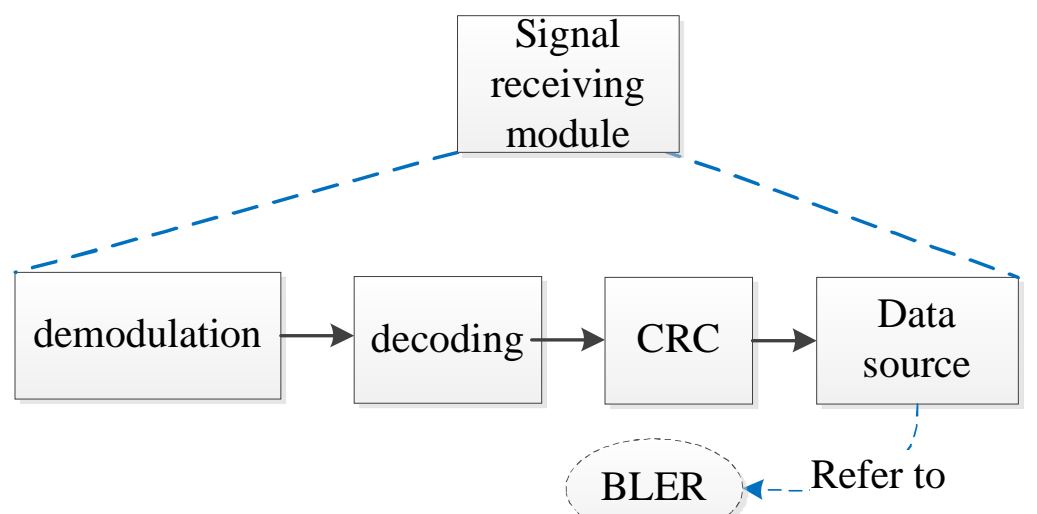

Fig 5. The contents of "Signal receiving module"

The specific parameters of the F-OFDM which introduced simplify above are described in this paragraph. The rate of FEC is $1 / 2$. The subcarrier spacing is $15 \mathrm{KHz}$ which is equivalent the OFDM symbol duration without GI is about $66.67 \mu \mathrm{s}$. The number of subcarrier is 600 , the oversampling size is 624 , that is to say the IFFT/FFT size is 1024. The CP (Cycle Prefix) selected as the GI (Guard Interval) and the period of $\mathrm{CP}$ is $16.67 \mu \mathrm{s}$, which is the max delay of the fading channel.

The PSD referred in Figure 3 are illustrated in Figure 6. The high side lobes of OFDM are eliminated by the sub band filter effectively.

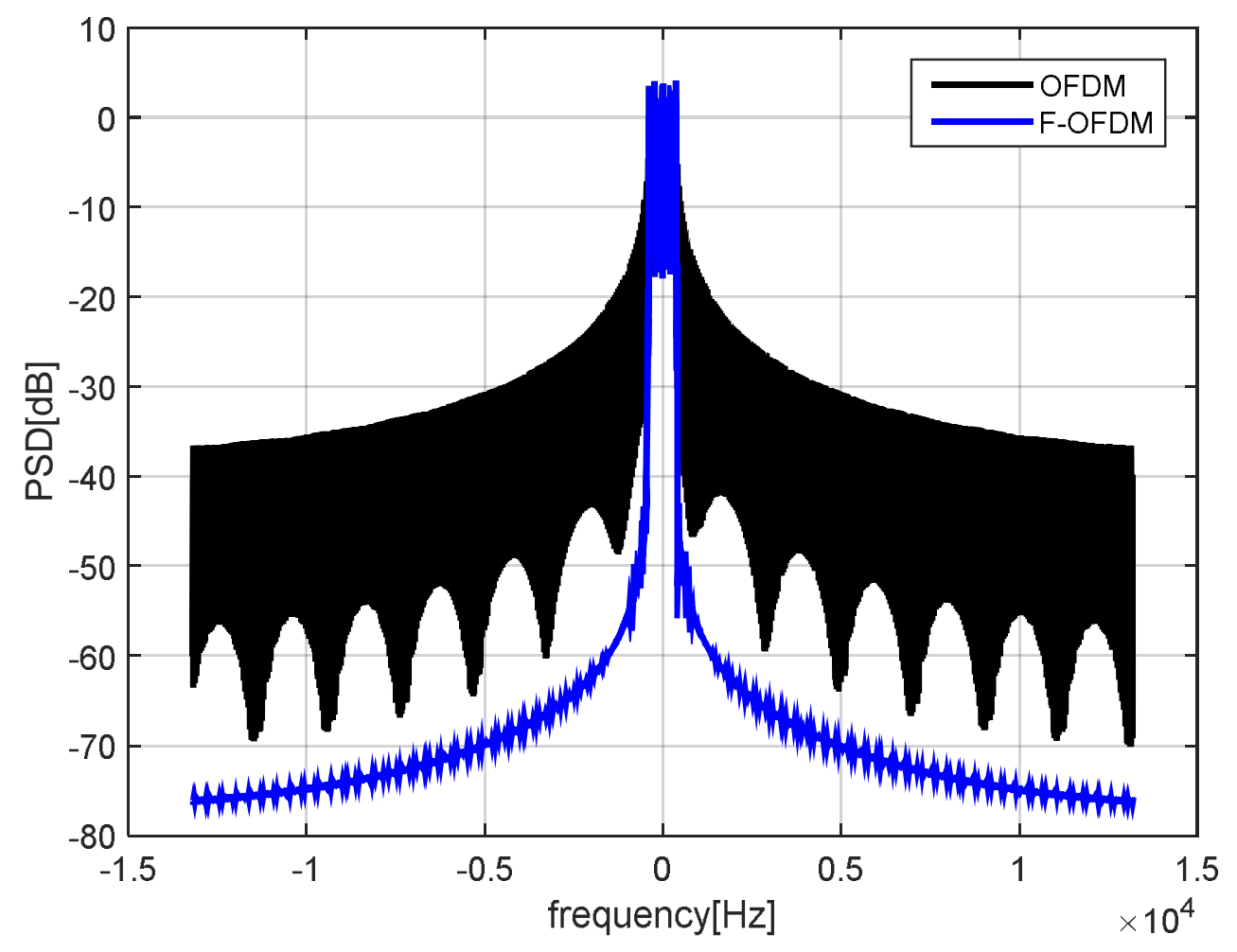

Fig 6. The PSD of OFDM and F-OFDM

The BLER referred in Figure 5 are drawn in the Figure 7. 


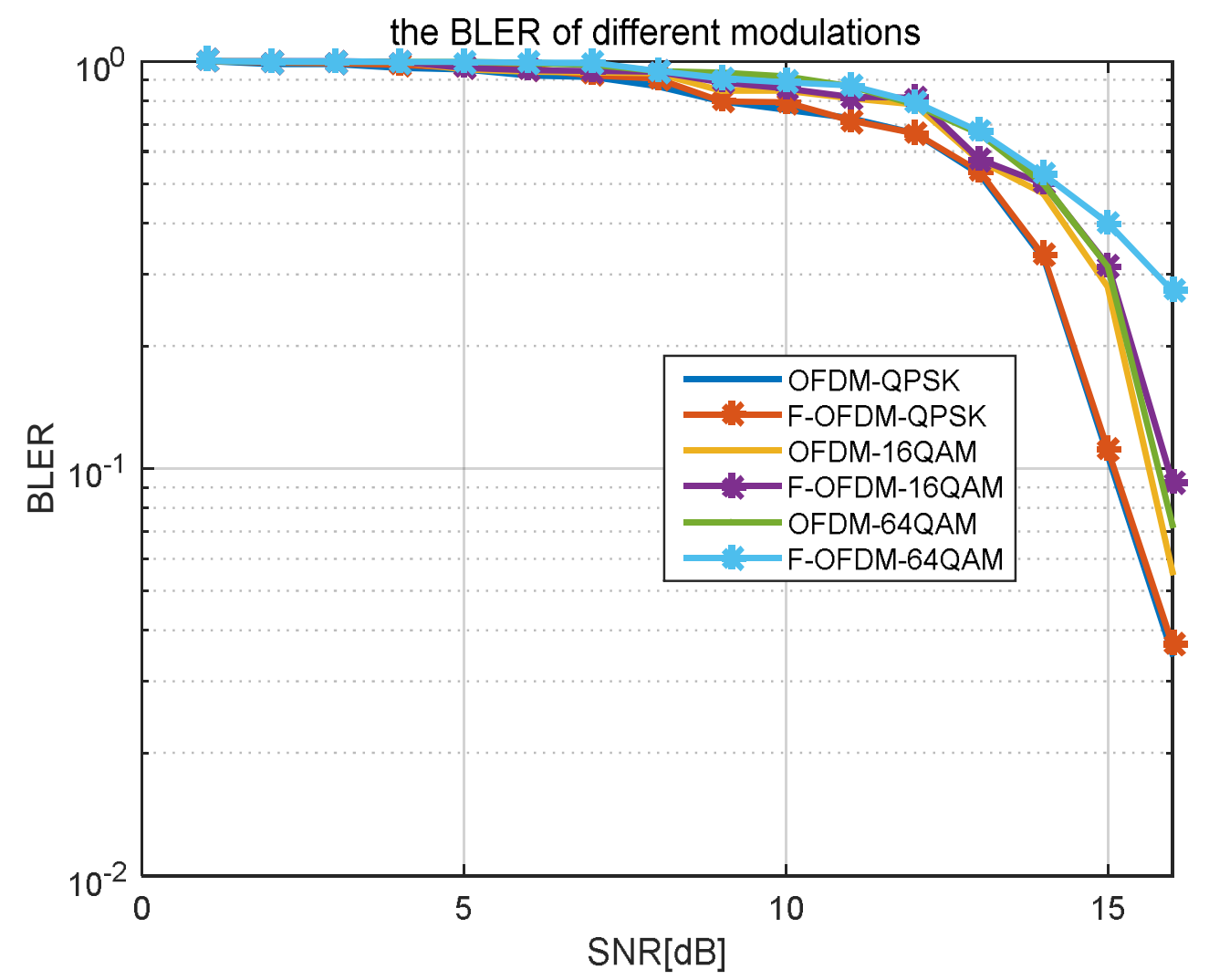

Fig 7. The BLER of OFDM and F-OFDM with different modulations

The better filtering operation is chosen according to the length of primary data by combining the $\gamma$ and $\lambda$, as shown in figure 8 (computational complexity considered only here).

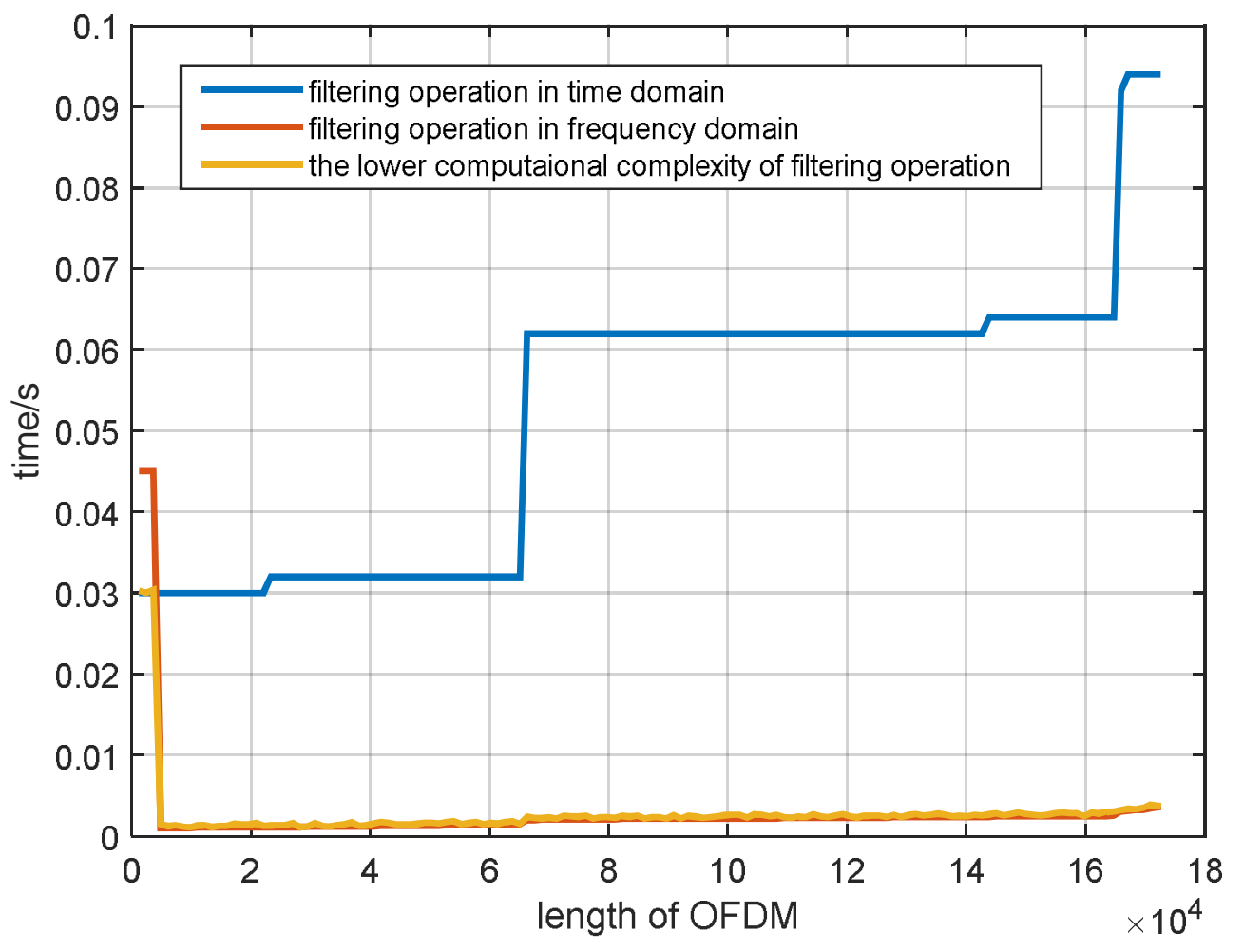

Fig 8. The running time of different filtering operation 
The total time saved for the whole F-OFDM link when always selects the lower computational filtering operation is drawn in figure 9.

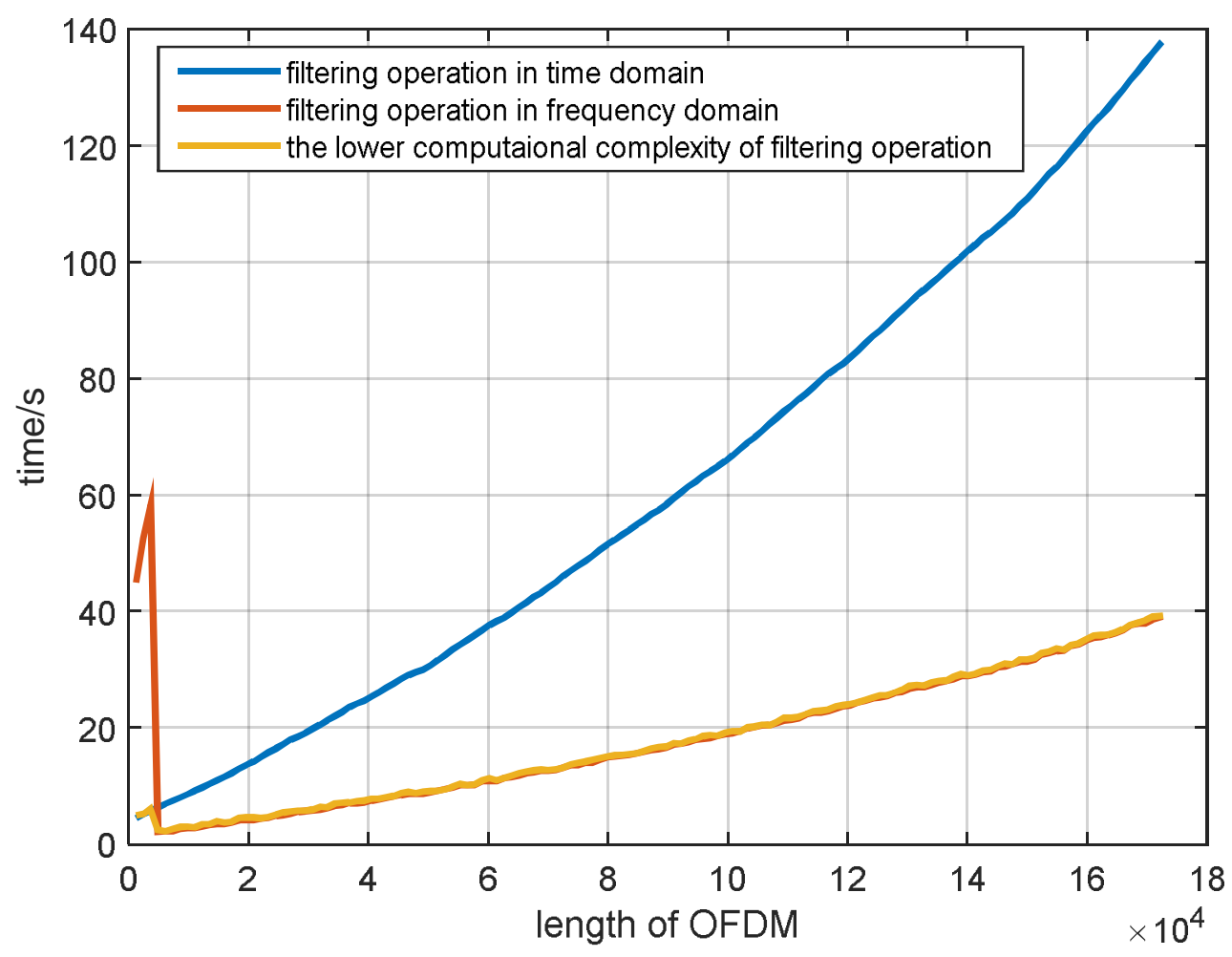

Fig 9. The running time of different filtering operation for whole F-OFDM downlink

\section{Conclusions}

The still existing disadvantage (high side lobes) of OFDM is settled by F-OFDM well. The BLER of F-OFDM in this paper can down below to 0.1 although is not good as BLER of [1], because of some skills are omited in the simplified block diagram of F-OFDM.

Lower computational complexity, lower space complexity or both the proper computational and space complexity can obtained by combining the $\gamma$ and $\lambda$. The lower computational complexity of filtering operation is selected when the less run time needed, just like Figure 8, 9 in this paper. This is quite useful for serially single progress and thread of simulation program. On the other hand, the lower space complexity of filtering operation can be obtained if the space is relatively rare. Generally speaking, the lower complexity can be gained by selecting the filtering operation in frequency domain, if the length of primary data to be filtered are large enough. The filtering operation in time domain may be selected when the data to be filtered are relatively small, and the lower space complexity may be obtained. In a words, the selection of better filtering operation depends on the demands for computational or space complexity.

\section{Acknowledgment}

Project supported by Chongqing integrated demonstration project (CSTC2013jcsf 10008).

\section{References}

[1] J. Adboli, M. Jia, and J. Ma, Filter OFDM: A new waveform for future wireless systems, IEEE International Workshop on Signal Processing Advances in Wireless Communications, 2015. 
[2] B. F. Boroujeny, OFDM versus filter bank multicarrier, IEEE Signal Processing Magazine, 2011, 28(3):92-112.

[3] M. Faulkner, The effect of filtering on the performance of OFDM systems, IEEE Transactions on Vehicular Technology, 2000, 49(5):1877-1884.

[4] T. Wild, F.Schaich, and Y.Chen, 5G air interface design based on universal filtered (UF-) OFDM, International Conference on Digital Signal Processing, 2014:699-704.

[5] V. Vakilian, T. Wild, F.Schaich, and S.Ten Brink, Universal-filtered multi-carrier technique for wireless systems beyond LET, IEEE Globecom Workshops, 2013:223-228.

[6] P. Pirinen, A Brief Overview of 5G Research Activities, International Conference on $5 \mathrm{~g}$ for Ubiquitous Connectivity, 2014:17-22.

[7] Information on http://www.innovateasia.com/5g/images/pdf/InnovateAsia-1st\%205G\%20Mobile\%20Algorithm\%20 Competition-F-OFDM.pdf

[8] BL. Floch , M. Alard , C. Berrou, Coded orthogonal frequency division multiplex, Proceedings of the IEEE, 1995, 83(6):982-996

[9] Information on https://inst.eecs.berkeley.edu/ ee123/sp15/docs/FastConv.pdf

[10] R. Van Nee, R. Prasad, OFDM for Wireless Multimedia Communications, Artech House, Boston, 2000 .

[11]E. Dahlman, S. Parkvall, and J. Skold, 4G: LTE/LTE-Advanced for Mobile Broadband, Second ed., Elsevier, Amsterdam, 2014. 